\title{
PROJETO E IMPLEMENTAÇÃO DE UM SISTEMA DE VISUALIZAÇÃO DE DADOS DE EPIDEMIAS OBTIDOS COLABORATIVAMENTE
}

\author{
Lucas C. de Almeida, Daniel S. do Prado, Lucas M. C. e Martins, \\ Daniel A. da Silva, Jonathas A. de Oliveira e Rafael T. de Sousa Júnior \\ Departamento de Engenharia Elétrica, Universidade de Brasília (UnB), Brasília-DF, Brasil
}

\begin{abstract}
RESUMO
A agregação e a visualização de dados constituem parte de um dos mais importantes campos de aplicação da Ciência de Dados na atualidade: o contexto da saúde coletiva. Entretanto, existem diversas fontes de dados, interesses econômicos e dificuldades técnicas no acesso confiável a esses dados. Este trabalho propõe e explora as escolhas tecnológicas para a implementação de um sistema no qual, de forma colaborativa, usuários fornecem informações de saúde, as quais são disponibilizadas por meio de painéis com escalabilidade no acesso e flexibilidade na análise, permitindo uma visão macro da situação. Também, com o uso dessas visualizações e das tecnologias de aplicações móveis, os usuários podem usufruir de serviços como acompanhamento de estado de saúde e envio de alertas de situações emergenciais.
\end{abstract}

\section{PALAVRAS-CHAVE}

Dados de Epidemia, Análise de Dados, Visualização de Dados, Ciência de Dados, Sistemas de Dados de Saúde Colaborativos, Saúde Coletiva

\section{INTRODUÇÃO}

Um dos maiores problemas dos centros urbanos é entender todo e qualquer fenômeno que se espalhe entre a população num curto prazo de tempo e tenha o potencial de atingir todos os cidadãos. Entre os diversos cenários possíveis, um em especial demanda acompanhamento e ajuste rápido de decisões: o contexto de uma epidemia. Em Lai, Yeung \& Celi (2020) e Ienca \& Vayena (2020), essa problemática é bem explorada, deixando claro o papel da tecnologia nesses cenários.

Essa situação foi verificada de forma dramática durante a pandemia da doença Covid-19. Sendo uma doença altamente contagiosa e sem vacina ou remédio cientificamente comprovado até a data deste trabalho (mais informações sobre a pandemia em WHO [2020]), análises de dados de localização dos casos verificados, data de notificação e de início dos sintomas, idade e até características físicas dos infectados se mostraram extremamente importantes para a compreensão da velocidade de espalhamento e do perfil de risco para a doença. Além disso, com o passar do tempo, determinadas regiões passaram a apresentar padrão de infecção diferente daquelas ao redor, exigindo medidas adicionais de distanciamento social sendo garantidas pelo próprio governo (um exemplo pode ser visto em Consultor Jurídico [2020]). Nenhuma dessas medidas e decisões fariam sentido se não existissem formas de mensurar os impactos e fazer comparações com o passado, e os sistemas de visualização, agregação e análise de Ciência de Dados são as ferramentas mais indicadas para esses estudos.

Todavia, mesmo sendo de amplo conhecimento a importância da tecnologia e da análise em situações de epidemias, ainda existem dificuldades globais de integração de bases de dados, de prontuários de pacientes, de acompanhamento de infectados, de receitas médicas entregues na saúde pública e privada, entre outros, todos relacionados com a dificuldade técnica em inserir no fluxo de trabalho dos profissionais de saúde métodos de coleta estruturada e limpezas de dados para posterior análise (em Santos et al. [2017] é possível ver um estudo sobre o tema). Diante dessa problemática, surge a opção de permitir ao usuário final (em oposição às unidades de saúde) o poder de fornecer dados, o que, quando amplamente aceito pela comunidade, resulta em uma base de dados altamente valiosa e importante na geração de métricas que 
exponham a situação de saúde geral da população. Ainda, ao mesmo tempo em que o usuário fornece, de boa vontade, dados próprios, também pode facilmente usufruir de serviços que o ajudem a manter e até melhorar a saúde, tais como diretrizes de proteção individual em relação ao distanciamento social indicado durante a epidemia de doenças contagiosas, serviços de acompanhamento de saúde (para pessoas doentes/infectadas) e até geração de histórico pessoal.

Neste trabalho, será apresentado um sistema dividido em módulos que, de forma resumida, permite aos usuários o fornecimento colaborativo de dados de saúde próprios e o acesso a serviços básicos como botão de emergência e verificação de diretrizes de proteção individual. A base de dados resultante é, então, consumida por uma aplicação que torna possível a análise desses dados tanto em visualização georreferenciada quanto em números e série histórica. Esse sistema, composto de interface de programação, aplicativo móvel e sistema gerenciador de banco de dados, foi posto em produção no ambiente de uma universidade e permitiu a implementação rápida do conceito de uma sala de monitoramento de saúde coletiva. O objetivo é mostrar o quanto a tecnologia pode contribuir, de forma simples e eficiente (desde que bem direcionada), para problemas tão atuais e relevantes como a saúde da população. Também, pretende-se que as lógicas de projeto usadas neste trabalho sejam referenciadas e sirvam de respaldo para a elaboração de outros projetos com objetivos semelhantes.

Este documento é composto por cinco seções, incluindo esta introdução. A próxima seção, de número 2 , trata de trabalhos relacionados. A Seção 3 especifica a proposta de sistema unindo tecnologias largamente utilizadas na indústria e que se integram de maneira descomplicada ao contexto dos usuários. A Seção 4 serve à discussão e demonstração de resultados obtidos na implementação do projeto com dados reais. As conclusões e trabalhos futuros encontram-se na Seção 5.

\section{TRABALHOS RELACIONADOS}

Importantes avanços têm sido feitos ao longo de anos para melhorar a captura e análise de dados em tempo real via aplicações mobile e outras técnicas do campo da mineração de dados. Esses avanços têm sido refletidos em estudos consistentes que permitem visualizar os benefícios práticos do uso de tais tecnologias e métodos no combate à proliferação de doenças contagiosas, tanto para fins de diagnóstico, como para o tratamento e monitoramento das mesmas. Dentre as possíveis visualizações, destacam-se as representações georreferenciadas, principal benefício do projeto descrito neste artigo.

Soluções de informação geográfica tais como o mapeamento online em tempo real (ou quase-real) de casos de doenças e de reações em mídias sociais à propagação de informações, juntamente com o mapeamento de risco preditivo, usando dados móveis do espaço e do tempo na locomoção de indivíduos, estão provando ser indispensáveis para o monitoramento e resposta oportuna e eficaz a epidemias, como visto no trabalho desenvolvido por Boulos \& Geraghty (2020). Os aplicativos sugeridos no trabalho referenciado visam captar dados de deslocamento dos usuários para estabelecer medições e avaliações do espalhamento geográfico de doenças contagiosas. Comparativamente, o sistema implementado no presente projeto tem potencial de contribuição mais amplo, visto que expande o auxílio no mapeamento do espalhamento geográfico de doenças para cobrir, também, o acompanhamento individual e a detecção e coleta de outros parâmetros valiosos como perfil de saúde dos usuários.

No trabalho desenvolvido em Arias-Carrasco et al. (2020), foi abordada a problemática da falta de ferramentas gratuitas e abertas para permitir que o público em geral possa visualizar em um dashboard, ou painel amigável para usuários comuns, dados geográficos relevantes sobre uma epidemia em destaque no momento. Sendo usada para estudos ou transparência ante a população, tal ferramenta pode ser de grande auxílio no estudo e combate a doenças e infecções. No trabalho referenciado, foi desenvolvido um painel específico para essa finalidade, a de prover visões simplificadas, intuitivas e amigáveis com gráficos sobre epidemias específicas. Os dados que alimentam o painel proposto no trabalho citado são provenientes do próprio público que fará o acesso ao painel, o que pode impactar na representatividade da informação. De forma diferente, no presente projeto, além de um dashboard amigável, os dados são captados diretamente dos usuários por meio de um aplicativo com padronização do formato das notificações. Portanto, a fonte de coleta de dados é o conjunto formado pelos próprios indivíduos afetados durante o surto epidemiológico de interesse, o que disponibiliza, para pesquisas científicas e discussões sobre políticas de combate, um importante viés técnico do cenário e do engajamento das pessoas. 
Embora considerem que não se pode substituir a vigilância tradicional, o trabalho apresentado por Mohanty, Chughtai \& Rabhi (2019) postula em seu trabalho que o uso de aplicações mobile pode ser complemento útil para a identificação antecipada de surtos epidemiológicos. No trabalho, foi realizado um levantamento de aplicativos móveis disponíveis em lojas virtuais e destinados a monitorar e controlar os impactos de doenças, além de prover dados para especialistas fazerem estudos e estabelecerem estratégias de ação no combate a doenças. $\mathrm{O}$ trabalho menciona a falta de alternativas de aplicativos destinadas para $\mathrm{o}$ público geral e disponíveis gratuitamente. Por fim, chegou-se à conclusão de que há um enorme potencial para novos aplicativos voltados para a área de saúde, especialmente no combate a doenças. A plataforma desenvolvida no presente projeto, de forma complementar, tenta estimular a linha de pesquisa relacionada a soluções colaborativas de vigilância de saúde coletiva, provendo funcionalidades que permitam ao usuário informar sobre sua saúde e obter auxílio quando necessário.

Dados obtidos por meio de aplicativos ou outros dispositivos tecnológicos também podem ser usados para alimentar um painel rico de informações relacionadas não somente a informações estatísticas, mas também com a alimentação de subsistemas de informações geográficas (ou Geographic Information System - GIS). Chiluba \& Dube (2020) focaram seus estudos em revisar e analisar os painéis geográficos disponíveis online para o mapeamento do surto de COVID-19, vivenciado no ano de 2020. Os painéis analisados utilizaram diferentes estratégias de representação geoespacial do impacto da doença. Dentre essas estratégias utilizadas pelas ferramentas analisadas, destacam-se: mapas coropléticos, circulares concêntricos; gráficos em pizza, buffer, overlay e aqueles com animações. Diferentemente do trabalho citado no parágrafo anterior, esse se dedica a fazer um levantamento das ferramentas analíticas com enfoque geoespacial. Por meio do levantamento de ferramentas já existentes, estabelece uma comparação qualitativa entre elas. O presente projeto se diferencia na medida em que, além de também possuir gráficos de um GIS, a fonte dos dados e a metodologia de coleta são bem conhecidas e estão à disposição para acesso e plotagem de outros gráficos através de uma interface programática.

Para ampliar o contexto dos trabalhos relacionados, o presente projeto tem como objetivo unir e explorar as possibilidades de cada tipo de solução abordada nos trabalhos citados e referente a cada etapa do processo de vigilância. Portanto, na seção a seguir, será apresentado o projeto e implementação de uma solução alternativa que integre as etapas de aquisição, armazenamento e análise de dados de saúde em uma única plataforma, auxiliando as equipes que elaboram políticas de combate e as de pesquisa e desenvolvimento que atuam na busca de tratamentos e elaboração de previsões e recomendações.

\section{ARQUITETURA PROPOSTA}

Este trabalho tem como proposta a implementação de uma plataforma capaz de facilitar a visualização e tomada de decisão baseada em dados de surtos sanitários por meio de informações fornecidas voluntariamente pelos usuários. Para esta finalidade, foi desenvolvido um conjunto de softwares preparados para coletar, processar e exibir registros de maneira facilitada para os operadores responsáveis pela solução.

Este sistema é composto por três diferentes serviços: Aplicativo móvel, API (Application Programming Interface) e Painel de Controle, o qual fornece e automatiza a análise e agregação de dados. Cada um, conjuntamente com as estratégias por trás das escolhas, serão explicados a seguir, sendo o foco deste artigo concentrado no valor adicionado ao trabalho dos profissionais de vigilância sanitária pela terceira parte da plataforma descrita na figura 1, a de análise e agregação de dados. Espera-se, também, que as motivações e os conceitos técnicos a serem abordados sirvam de guia, respaldo e inspiração para iniciativas com temática semelhante.

\subsection{Aplicativo Móvel}

A primeira preocupação do projeto era referente à forma de aquisição dos dados e viabilização do recebimento de alertas, funcionalidade que será explicada posteriormente. Antes de qualquer análise, considerando se tratar de um projeto que depende de dados coletados voluntariamente, é necessário entender a estratégia adotada para a interação com os usuários. No caso, era necessário escolher uma tecnologia que fosse popular o suficiente para atingir qualquer interessado, mas que não se tornasse restritiva a ponto de limitar quaisquer benefícios que pudessem ser revertidos para a comunidade engajada. Ainda, era necessário 
que essa tecnologia fosse altamente escalável e que permitisse o acesso a informações que não têm distribuição trivial, ou seja, que não podem ser fornecidas de forma manual, simples e descomplicada pelas pessoas, como a localização geográfica. Complementarmente, também é importante destacar que a escolha da tecnologia deveria privilegiar a privacidade dos usuários, de forma que dados como a localização fossem coletados apenas mediante autorização explícita do usuário no momento do cadastro.

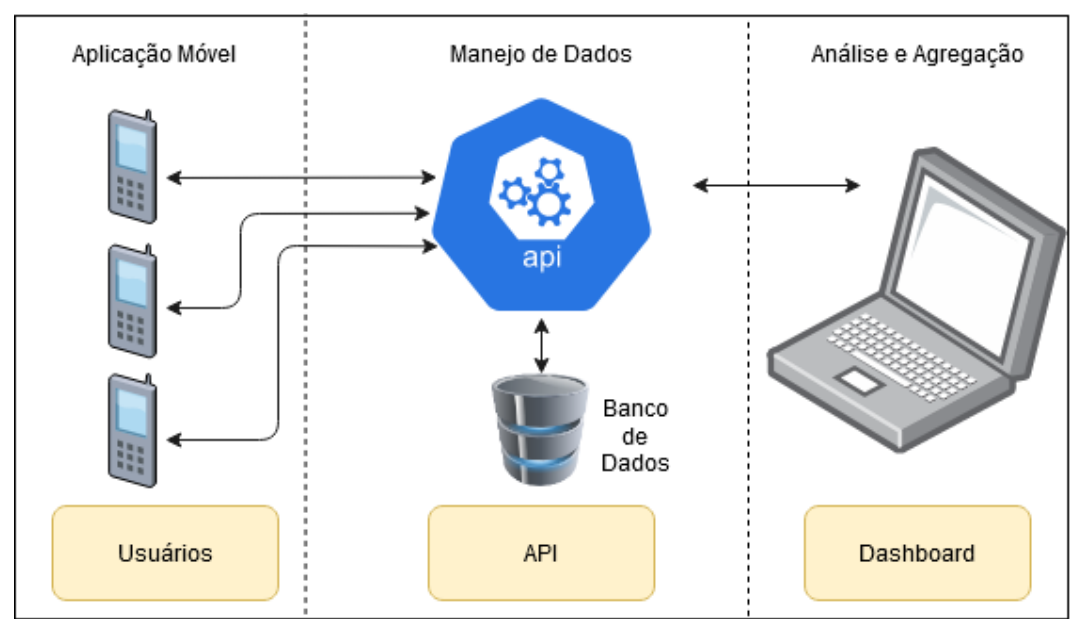

Figura 1. Estrutura da plataforma separada em módulos. Cada módulo é responsável por um serviço

Esse conjunto de requisitos levou à escolha das aplicações móveis como ponto de coleta de dados. Os aparelhos celulares da atualidade têm, de forma predominante, acesso à Internet e a possibilidade de instalação rápida e gratuita de programas com visual e interação bastante orientados aos usuários finais, além de poderem ser instalados sem restrição de quantidade, conforme o paradigma da aplicação nativa apresentada em Huy \& vanThanh (2012). Em resumo, o fato de que cada aparelho móvel executa localmente um programa e consome os serviços de uma aplicação em nuvem tornam a experiência do usuário intuitiva, independente e altamente escalável, conceitos alinhados com o tipo de arquitetura proposta para este trabalho, a de orientação a serviços. Também, os smartphones são parte do topo da lista de equipamentos mais populares no mundo, conforme previsões e tendências em Cisco (2020). Assim, o módulo mobile se torna parte fundamental para um projeto com ambições de colaboração voluntária. Nele, os usuários deverão poder se cadastrar na plataforma proposta e fornecer dados sobre seu estado de saúde e perfil (ações primordiais para o funcionamento da análise de dados). Também, o aplicativo enviará, periodicamente e de forma autônoma, a localização do usuário, facilitando todo o processo de vigilância. Essa última funcionalidade também se beneficia bastante da escolha pelas aplicações móveis, pois ainda que não seja comum pessoas se locomoverem com rastreadores de localização geográfica, os aparelhos smartphones disponibilizam não apenas essa funcionalidade, como também poder de processamento relevante, possibilidade de automações diversas, tarefas em segundo plano e interface de usuário bastante amigável.

É importante destacar, sobre a localização, que essa é descaracterizada nos painéis. Para manter a privacidade dos usuários, a visualização não permite saber a identidade de cada pessoa no mapa, a não ser que se trate de operador com autorização (nível de acesso) para tal finalidade, o que se pretende, num momento inicial, ser bastante restrito. A ideia é que essa possibilidade exista apenas para os profissionais que farão o atendimento a alertas e o acompanhamento de casos de infecções. O acesso com menos privilégios, ou seja, que não permite verificar dados pessoais, seria utilizado, por exemplo, por pesquisadores e administradores, os quais não irão interagir com os voluntários, mas usarão os dados globais para tomada de decisão e estudo.

\subsection{API}

Conforme comentado anteriormente, a escolha de uma aplicação móvel tem a vantagem de ser altamente escalável quando o projeto da plataforma é feito baseado na divisão em serviços. Contudo, importa não apenas que as aplicações móveis tenham essa escalabilidade, mas que os programas que irão requisitar dados 
e realizar análises também sejam independentes e tenham liberdade de trabalho. Esses conceitos direcionam um projeto para a diferenciação entre quem provê e administra dados e quem insere e/ou manipula a informação. Todavia, em aplicações disponíveis por intermédio da Internet, ainda existem considerações relacionadas à segurança, à forma e também à extensão do acesso de cada módulo aos dados. Também, a forma de entrega desses dados pode onerar bastante o desenvolvimento de software justamente devido ao fato de que vários sistemas operacionais de várias plataformas, com representações diferentes de dados (conceito denominado de "endianness", explicado em Google Patents [2014]), estão acessando e inserindo informações na mesma base, sem protocolo ou algoritmo algum que garanta a isonomia das representações. Por fim, a escolha da interface para dados levanta outras problemáticas, e entre elas uma com natureza relacionada ao nível de acesso por função: não existe a necessidade de cada exemplar da aplicação ter acesso integral à base (assim como um usuário voluntário não deve ter nível de acesso máximo aos dados do projeto e nem ter liberdade de inserção em formatos diferentes dos que a aplicação espera), justamente porque cada instância é executada em um contexto privado (o celular do usuário). Essa enumeração de problemas introduz a necessidade das chamadas API's.

Uma API ou, traduzido do inglês, uma Interface de Programação de Aplicação, define um conjunto de chamadas que podem ser usadas de forma organizada por outros programas para integrar diferentes módulos de software. Cada chamada é projetada e definida pelos programadores de forma que as problemáticas levantadas são obrigatoriamente observadas e tratadas para a implementação:

1. Em relação à forma, os programadores precisam, necessariamente, definir a formatação e a filtragem dos dados de cada possível chamada de uma função da API antes de desenvolvê-la e disponibilizá-la. Isso implica que os dados são disponibilizados apenas segundo a visão projetada na interface da API;

2. Em relação à representação isonômica dos dados, problema especialmente importante no contexto de aplicações dependentes da Internet, uma API sempre é desenvolvida baseada num protocolo definido por padrões de Indústria. Esse protocolo, entre outras finalidades, deve garantir a conversão correta de dados de forma transparente para as aplicações finais. Nesse caso, o protocolo da camada de aplicação da pilha TCP/IP da Internet que define o formato das mensagens e chamadas é o Hypertext Transfer Protocol (HTTP), definido em Fielding \& Reschke ( 2014). Assim, independentemente da plataforma e software, existe um compromisso de adequação da conversa ao que rege o protocolo HTTP, garantindo a igualdade do dado independentemente de plataforma;

3. Em relação à extensão e nível de acesso, cada chamada possível da API retorna (ou insere) somente os dados definidos durante o desenvolvimento. Portanto cada módulo, de forma efetiva, trabalha em escopos bem traçados e coerentes de acesso à base de dados.

Portanto, para permitir a independência entre os módulos de análise e de interação dos usuários, garantir as boas práticas de um projeto separado por serviços com contextos bem definidos e prover alta escalabilidade no manejo dos dados, desenvolveu-se uma API baseada no protocolo HTTP com métodos que regulam o acesso aos dados e a inserção de informações no banco de forma coerente. Por todos os motivos e análises dispostos, o projeto e desenvolvimento de uma API se mostra como escolha primordial para qualquer projeto baseado em interações voluntárias e aquisição de dados de usuários finais por meio da Internet.

\subsection{Painel de Controle}

Com o foco exclusivo em visualização e geração de valor para os dados, um painel de controle (ou dashboard, termo vindo do inglês e usado de forma global) foi desenvolvido para agregar os dados que são enviados pelos usuários para a API e apresentá-los de maneira que seja possível tanto ter a noção ampla do conjunto de dados, quanto acompanhar mudanças quase no mesmo momento em que ocorrem. Esse é o módulo responsável por facilitar a vigilância sanitária e aproximar o acompanhamento feito pelos profissionais de saúde com os usuários finais.

Esse dashboard está dividido em duas seções, as quais são descritas a seguir. 


\subsubsection{Usuários Cadastrados}

Nesta parte do painel, é possível visualizar, num mapa ou numa lista, todos os usuários cadastrados na plataforma. Utilizou-se um sistema de cores que servem para distinguir visualmente o perfil de risco dos usuários: os que apresentam a cor verde são os usuários com menos 50 anos de idade e que não reportaram nenhuma comorbidade; amarelo representa aqueles com idade acima de 50 anos ou que apresentam comorbidades; e vermelho simboliza os que apresentam idade acima de 50 anos e, ao mesmo tempo, possuem comorbidades. Entenda-se comorbidades como condições de saúde preexistentes que possam se somar a outras doenças e/ou serem facilitadoras da infecção de novas doenças, como por exemplo obesidade e hipertensão (conforme definição disponível em Valderas et al. [2009]).

Com a fácil distinção entre os diferentes grupos, simplifica-se o direcionamento da atenção aos usuários, principalmente considerando a utilização desta aplicação por profissionais de saúde, comentado melhor posteriormente.

Importa, ainda, destacar que a visualização proposta tem outras funcionalidades que extrapolam o contexto de uma única epidemia ou doença. Como exemplo, dada a definição de comorbidade citada e a separação por faixas etárias, se torna possível conduzir estudos para determinação de fatores locais que possam estimular a manifestação coletiva dessas comorbidades e tomar decisões que afetem diretamente a saúde das pessoas.

\subsubsection{Alertas}

Essa aba dispõe a visualização facilitada dos alertas gerados pelos usuários da plataforma. A visualização pode ser em forma de lista ou mapa. Também é utilizado um sistema de cores semelhante ao de usuários cadastrados, contudo, nesse caso as cores estão dispostas de acordo com o sintoma reportado, não com as características dos usuários. Caso seja reportado apenas um dos sintomas comuns a várias doenças como dor de cabeça, febre ou espirro, por exemplo, a cor verde é apresentada. A cor amarela é apresentada caso sejam reportados dois destes tipos de sintomas, e a cor vermelha, um conjunto de três ou mais sintomas ou se o usuário reportar sintomas críticos e bastante relacionados à doença Covid-19, tais como falta de paladar, olfato ou ainda falta de ar.

O desenvolvimento inicial da aplicação foi motivado pela pandemia da doença Covid-19. Porém a aplicação final e modelo de projeto é genérica o suficiente para ser estendida para a visualização de quaisquer dados que envolvam doenças contagiosas e casos relacionados à vigilância sanitária.

Outro ponto importante a ser mencionado é que, como o usuário compartilha com a aplicação dados pessoais e sobre sua saúde, é possível a inclusão de profissionais da saúde no processo de vigilância que realizem intervenções mais direcionadas e pessoais para cada usuário. Essa situação é especialmente relevante em casos de epidemia, em que o acompanhamento dos infectados é de suma importância (Daumas et al. [2020] discorre sobre o tema). Portanto, esses profissionais poderiam, com o apoio da plataforma, direcionar esforços e atender, à distância, pessoas em situações de vulnerabilidades diversas.

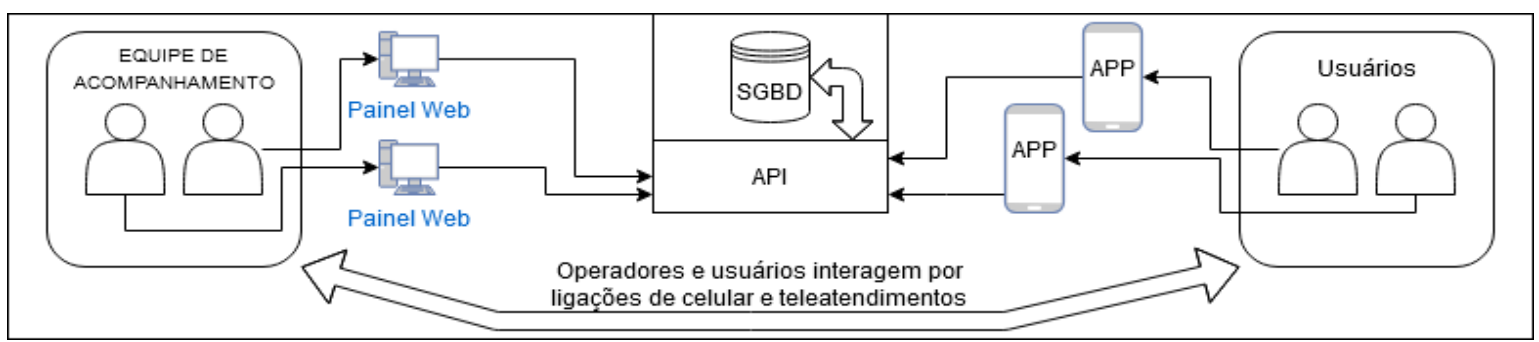

Figura 2. Caminho dos dados e funcionalidades para a plataforma proposta no contexto de uma sala de vigilância epidemiológica. O termo SGBD se refere a um Sistema Gerenciador de Banco de Dados

É interessante notar, também, que os alertas emitidos pelos usuários contém sua localização geográfica, facilitando o monitoramento geográfico de informações por parte do profissional que estiver de prontidão, de forma que é possível observar não apenas os grupos de risco, mas também mapas de calor dos pedidos de socorro e do número de usuários cadastrados, visão importantíssima para a administração da saúde de uma grande cidade, por exemplo, que pode remanejar com mais eficiência ambulâncias e cruzar dados de tráfego para redução do tempo de espera. Na figura 2 é exibido um esquema do uso pretendido para a solução final no contexto de uma sala de vigilância. 


\section{TESTES E RESULTADOS}

Os testes do sistema foram realizados em um ambiente quase controlado: o sistema foi posto em produção em ambiente real de uso, mas o grupo de usuários com acesso ao sistema foi restrito. Mais especificamente, 22 pessoas se candidataram para testar a plataforma. Esse grupo foi formado por discentes e docentes de uma universidade, sendo variado em relação aos perfis de risco e condições de saúde preexistentes.

Com os módulos de software em produção, foi pedido que os usuários fizessem o cadastro na plataforma com dados fictícios e enviassem alertas de saúde em momentos aleatórios. A figura 3 mostra a visão da distribuição georreferenciada dos cadastrados com as respectivas cores de acordo com o perfil de cada usuário. Testes também foram feitos tanto para estressar a visão de alertas e verificar se a qualidade dos pedidos no mapa permitia adequada visualização georreferenciada, quanto para validar o conceito de que um grupo de monitoramento poderia atuar de forma concisa, eficiente, e em tempo hábil. É interessante observar que a precisão da localização coletada automaticamente poderia variar com a qualidade da conexão do usuário com a internet e com o sinal de GPS (traduzido do inglês, Sistema de Posicionamento Global), especialmente em locais confinados e sem janelas. Também, a confiabilidade na chegada dos alertas foi satisfatória (desde que o usuário tivesse acesso à internet através do celular), criando uma fila a qual permitia aos operadores entrar em contato, pelo número telefônico, com cada usuário que tivesse feito o pedido (note-se que apenas os operadores da sala de atendimento que têm acesso aos dados pessoais dos voluntários, profissionais com outras finalidades têm acesso apenas à massa de dados descaracterizados). A figura 4 mostra a visão que uma equipe de monitoramento tem dos usuários pedindo socorro por intermédio do aplicativo.

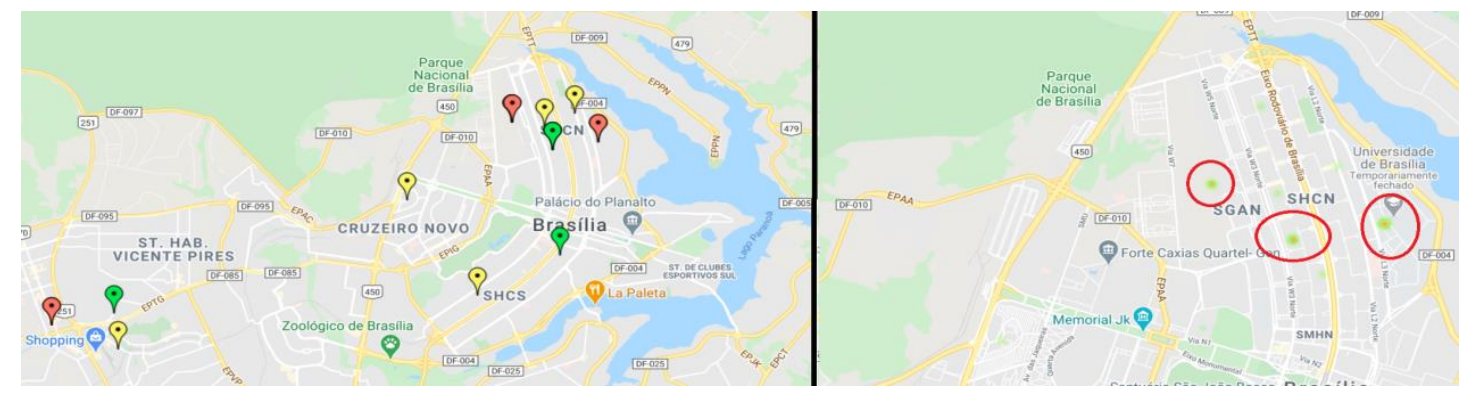

Figura 3. À esquerda, os usuários caracterizados pelo respectivo perfil de risco. À direita, mapa de calor com a aglutinação de alertas. As imagens são processadas pela API de mapas da empresa Google ([Google Maps Platform, 2020])

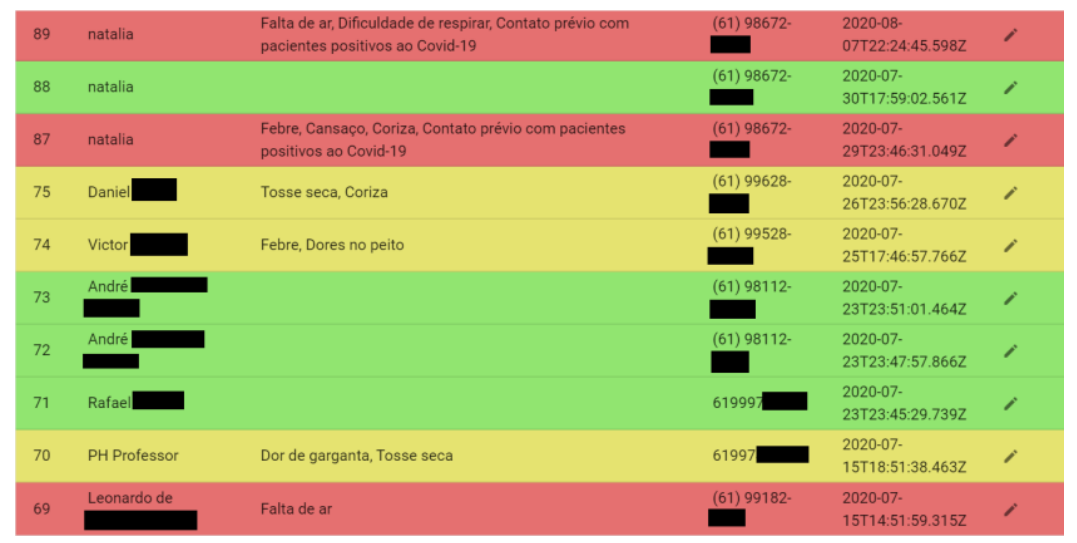

Figura 4. Painel com lista dos pedidos de socorro. Todos os dados são fictícios, contudo, os números de telefone foram censurados caso correspondam a algum número real. Os sobrenomes também foram censurados onde aplicável. Os alertas sem descrição significam que a pessoa está sem nenhum sintoma 
O valor percebido pela agregação dos dados e visualização georreferenciada, bem como o poder da colaboração dos usuários e a facilidade na implantação de um serviço de pedido de socorro ficam bastante evidentes ao avaliar as figuras 3 e 4 . O produto final resulta em todo aparato lógico necessário para o funcionamento adequado de uma sala de monitoração de saúde coletiva, iniciativa que se mostra cada vez mais importante quanto mais relevantes se tornam os riscos sanitários das grandes aglomerações urbanas. Com o uso do sistema proposto, uma equipe enxuta se torna capaz de prestar serviços de direcionamento de socorro e analisar de forma profunda a saúde de uma comunidade grande.

\section{CONCLUSÕES E TRABALHOS FUTUROS}

A pandemia da doença Covid-19 apresentou-se repentinamente como um problema de saúde pública em todo o mundo, tendo impactado significativamente a vida da maior parte da população e demandado decisões nos mais diversos segmentos da sociedade, em especial na saúde.

Esse contexto motivou este trabalho, o qual apresenta o projeto e teste de uma plataforma capaz de apoiar forças de trabalho inseridas em cenários não só de exposição a doenças contagiosas, como uma epidemia, mas de incidentes de saúde gerais. A solução proposta atua em duas frentes: por um lado, é um canal que permite às pessoas informarem suas condições de saúde e pedirem socorro quando necessário, e por outro, permite que autoridades e profissionais de saúde acompanhem em tempo quase-real e de forma facilitada a saúde coletiva. É importante destacar que este trabalho tem como premissa a colaboração dos usuários no fornecimento de dados fiéis para permitir a inferência e conclusão da situação do contágio da doença na região.

A ferramenta apresentada é um sistema distribuído no qual se consideram as boas práticas de projeto de forma que permita aos módulos do sistema serem escaláveis para poder manipular grandes volumes de dados acessíveis de qualquer lugar por intermédio da Internet. Portanto, o projeto e funcionamento dos três módulos construídos foram apresentados neste trabalho: a lógica do aplicativo móvel com foco nos usuários finais, o painel de controle com foco na equipe de monitoramento e nos pesquisadores e/ou tomadores de decisão, e a API que permite a comunicação entre ambos contextos.

O sistema foi aplicado em caráter experimental numa comunidade acadêmica e foi possível perceber, de forma visual, locais e pessoas que necessitavam de mais atenção, assim como localizar pedidos de socorro feitos pelos usuários e dispor de filas de notificações em ordem cronológica e com diferenciação de prioridade para melhor gestão dos atendimentos.

Como trabalho futuro, vislumbra-se a aplicação de modelos de análise de dados, de forma que seja possível realizar predições sobre a evolução da doença contagiosa na região monitorada. Outro ponto a ser explorado é a possibilidade de integrar os dados fornecidos de forma colaborativa pelo sistema apresentado neste trabalho com informações obtidas de outras fontes como, por exemplo, dados de saúde pública fornecidos por organizações de saúde do poder público e dados coletados em redes sociais. Ainda, a integração da aplicação com dispositivos pessoais de monitoramento de saúde pode permitir a fácil disponibilização de serviços de saúde para diversas situações, até de naturezas críticas, como o pedido automático de socorro para acidentados em quedas, ataques do coração e colisões de veículos, por exemplo.

\section{AGRADECIMENTOS}

Os autores agradecem o apoio das Agências brasileiras de pesquisa, desenvolvimento e inovação CNPq (Projetos INCT SegCiber 465741/2014-2, PQ-2 312180/2019-5 e LargEWiN BRICS2017-591), CAPES (Projetos FORTE 23038.007604/2014-69 e PROBRAL 88887.144009/2017-00) e FAPDF (Projetos UIoT 0193.001366/2016 e SSDDC 0193. 001365/2016), bem como o suporte do Laboratório LATITUDE/UnB (Projeto SDN 23106. 099441/2016-43), a cooperação com o Ministério da Economia (TEDs DIPLA 005/2016 e ENAP 083/2016), o Gabinete de Segurança Institucional da Presidência da República (TED 002/2017), a Advocacia-Geral da União (TED 697.935/2019) e o Conselho Administrativo de Defesa Econômica (TED 08700.000047/2019-14). 


\section{REFERÊNCIAS}

Arias-Carrasco, R. et al., 2020. OUTBREAK: A user-friendly georeferencing online tool for disease surveillance. arXiv:2004.10490v1

Chiluba, B. \& Dube, G., 2020. Descriptive Review of Epidemiological Geographic Mapping of Coronavirus Disease 2019 (COVID-19) on the Internet. In Biomedical and Biotechnology Research Journal (BBRJ), Vol. 4, No. 2, pp. 83-89.

Mohanty, B., Chughtai, A. \& Rabhi, F., 2019. Use of Mobile Apps for epidemic surveillance and response - availability and gaps. In Global Biosecurity, Vol. 1, No. 2, pp. 37-49. DOI: 10.31646/gbio.39

Boulos, M. K. \& Geraghty, E., 2020. GeGeographical tracking and mapping of coronavirus disease COVID-19/severe acute respiratory syndrome coronavirus 2 (SARS-CoV-2) epidemic and associated events around the world: how 21st century GIS technologies are supporting the global fight against outbreaks and epidemics. In International Journal of Health Geographics, Vol. 19, No. 8. DOI: 10.1186/s12942-020-00202-8

Cisco, 2020. Cisco Annual Internet Report (2018-2023) White Paper [Online]. Available at: https://www.cisco.com/c/en/us/solutions/collateral/executive-perspectives/annual-internet-report/white-paper-c11741490.html (Accessed: 15 Aug 2020)

Consultor Jurídico, 2020. Bruno Covas estende quarentena para 15 de junho na capital paulista [Online]. Available at: https://www.conjur.com.br/2020-mai-30/quarentena-estendida-15-junho-capital-paulista (Accessed: 15 Aug 2020)

Daumas, Regina P. et al., 2020. O papel da atenção primária na rede de atenção à saúde no Brasil: limites e possibilidades no enfrentamento da COVID-19. In Cad. Saúde Pública, Vol. 36, No. 6, pp. e00104120. DOI: 10.1590/0102$311 \times 00104120$.

Fielding, R., \& Reschke, J., 2014. Hypertext Transfer Protocol (HTTP/1.1): Semantics and Content (RFC 7231) [Online]. Internet Engineering Task Force (IETF). Available at: https://tools.ietf.org/html/rfc7231 (Accessed: 15 Aug 2020)

Google, 2014. Google Maps Platform [Online]. Available at: https://cloud.google.com/maps-platform?hl=pt-br (Accessed: 15 Aug 2020)

Google, 2020. Multiple endianness compatibility - US Patent US10163467B2 [Online]. Available at: https://patents.google.com/patent/US10163467B2/en (Accessed: 15 Aug 2020)

Huy, N. P., \& vanThanh, D., 2012. Evaluation of mobile app paradigms. Proceedings of the 10th International Conference on Advances in Mobile Computing \& Multimedia (MoMM '12). Bali, Indonesia, pp. 25-30. DOI: $10.1145 / 2428955.2428968$.

Ienca, M., Vayena, E., 2020. On the responsible use of digital data to tackle the COVID-19 pandemic. In Nat Med, Vol. 26, pp. 463-464. DOI: 10.1038/s41591-020-0832-5

Lai, Y., Yeung, W. \& Celi, L. A., 2020. Urban Intelligence for Pandemic Response: Viewpoint. In JMIR Public Health Surveill, Vol. 6, No. 2, pp. e18873. DOI: 10.2196/18873

Santos, Alaneir de Fátima dos et al., 2017. Incorporação de Tecnologias de Informação e Comunicação e qualidade na atenção básica em saúde no Brasil. In Cad. Saúde Pública, Vol. 33, No. 5, e00172815. DOI: $10.1590 / 0102-311 \times 00172815$.

Valderas, J. M. et al., 2009. Defining comorbidity: implications for understanding health and health services. In The Annals of Family Medicine, Vol. 7, No. 4, pp. 357-363. DOI: 10.1370/afm.983

World Health Organization (WHO), 2020. Q\&A on coronaviruses (COVID-19) [Online]. Available at: https://www.who.int/emergencies/diseases/novel-coronavirus-2019/question-and-answers-hub/q-a-detail/q-acoronaviruses (Accessed: 15 Aug 2020) 